\title{
REX: A Randomized EXclusive Region Based Scheduling Scheme for mmWave WPANs with Directional Antenna
}

\author{
Lin X. Cai, Student Member, IEEE, Lin Cai, Member, IEEE, Xuemin (Sherman) Shen, Fellow, IEEE, \\ and Jon W. Mark, Life Fellow, IEEE
}

\begin{abstract}
Millimeter-wave (mmWave) transmissions are promising technologies for high data rate (multi-Gbps) Wireless Personal Area Networks (WPANs). In this paper, we first introduce the concept of exclusive region (ER) to allow concurrent transmissions to explore the spatial multiplexing gain of wireless networks. Considering the unique characteristics of mmWave communications and the use of omni-directional or directional antennae, we derive the ER conditions which ensure that concurrent transmissions can always outperform serial TDMA transmissions in a mmWave WPAN. We then propose REX, a randomized ER based scheduling scheme, to decide a set of senders that can transmit simultaneously. In addition, the expected number of flows that can be scheduled for concurrent transmissions is obtained analytically. Extensive simulations are conducted to validate the analysis and demonstrate the effectiveness and efficiency of the proposed REX scheduling scheme. The results should provide important guidelines for future deployment of mmWave based WPANs.
\end{abstract}

Index Terms-Resource management, exclusive region, service scheduling, spatial multiplexing gain, mmWave WPAN.

\section{INTRODUCTION}

$\mathbf{T}$ HE spectrum between $30 \mathrm{GHz}$ and $300 \mathrm{GHz}$ is referred to as the millimeter wave (mmWave) band because the wavelengths for these frequencies are about one to ten millimeters. The FCC has recently allocated the $57-64 \mathrm{GHz}$ mmWave band for general unlicensed use, which opens a door for very high data rate wireless applications over the $7 \mathrm{GHz}$ unlicensed band. The IEEE 802.15.3c has recently been formed to develop a mmWave-based alternative physical layer (PHY) for the existing 802.15.3 Wireless Personal Area Networks (WPANs) standard. The mmWave communications have many salient features. First, it is anticipated to achieve very high data rate (multi-Gbps), so it will enable many killer applications such as IPTV/VoD, 3D gaming, intelligent transportation systems, etc. These applications require not only high data rate, but also stringent QoS, in terms of delay,

Manuscript received May 14, 2007; revised July 15, 2007; accepted July 25, 2007. The associate editor coordinating the review of this paper and approving it for publication was X.-G. Xia.

L. X. Cai, X. Shen, and J. W. Mark are with the Centre for Wireless Communications, Department of Electrical and Computer Engineering, University of Waterloo, Waterloo, ON N2L 3G1, Canada (e-mail: \{lcai, xshen, jwmark\}@bbcr.uwaterloo.ca).

L. Cai is with the Department of Electrical and Computer Engineering, University of Victoria (e-mail: cai@uvic.ca).

This work has been supported by the Natural Sciences and Engineering Research Council (NSERC) of Canada under Grant No. RGPIN7779.

Digital Object Identifier 10.1109/TWC.2010.01.070503 jitter, and loss. Second, mmWave systems can coexist well with existing wireless communication systems, such as $\mathrm{WiFi}$ (IEEE 802.11), cellular systems, and Ultra WideBand (UWB) systems, because of the large frequency difference. Third, oxygen absorption peaks at $60 \mathrm{GHz}$, so the transmission and interference ranges of mmWave communications are small, which allows highly dense deployment of mmWave WPANs. In addition, since the mmWave signal degrades significantly when passing through walls and over distances, this will help to ensure the security of the content.

Although mmWave prototype chipsets have been emerging [1], their performance in a networked environment is still an open area beckoning for further investigation. To ensure the success of mmWave based WPANs, how to efficiently and effectively allocate resource for co-existing mmWave devices is a critical issue, which is the main focus of this paper.

In this paper, we first investigate the unique characteristics of mmWave communications, the appropriate medium access techniques, and network architecture for mmWave based WPANs. We then identify the key opportunities and challenges in resource management of mmWave WPANs, and propose REX, a randomized exclusive region (ER) based scheduling scheme to explore the spatial multiplexing gain in mmWave WPANs. The basic concept of REX is: each flow has an ER around the receiver, and the senders of all flows transmitting concurrently should be outside the ERs of other flows to ensure that concurrent transmissions are favorable.

The main contributions of the paper are four-fold. First, to the best of our knowledge, the paper is one of the first to systematically study the resource management issues for mmWave based WPANs. Second, we propose how to allow concurrent transmissions appropriately to explore the spatial multiplexing gain in mmWave WPANs, and derive the sufficient conditions to ensure that concurrent transmissions are favorable in terms of per flow throughput and network throughput, considering both omni-(directional) and directional antennae. Third, optimal scheduling for peer to peer concurrent transmissions is known to be NP-hard [2], [3]. In traditional scheduling problems, the utility (e.g., throughput) obtained per unit resource (e.g., bandwidth $\times$ time slot) is deterministic; here, utility is variable according to channel data rate, network topology, user deployment, transmission power, cross-correlations of interfering signals, and the scheduling decision itself. Since the optimal scheduling problem is dif- 
ficult to solve in real time, we propose the REX scheme as the first step to explore the spatial multiplexing capacity of mmWave WPANs. Finally, given the ER condition, we analytically investigate the network performance in terms of the expected number of concurrent transmissions. Extensive simulations have demonstrated the accuracy of the analysis and the efficiency of the proposed scheduling scheme.

The remainder of the paper is organized as follows. In Sec. II, we present the channel characteristics of mmWave communications and the architecture design of mmWave based WPANs. In Sec. III, we derive the exclusive regions considering both omni- and directional antennae. The proposed REX scheme is presented in Sec. IV-A, and its performance is analyzed in Sec. IV-B. Simulation results are presented in Sec. V, followed by the related work in Sec. VI. Concluding remarks and future work are given in Sec. VII.

\section{System Model}

\section{A. mmWave Channel Characteristics and Multiple Access}

The main characteristics of mmWave communications are short wavelength/high frequency, large bandwidth and high interaction with atmospheric constituents. For mmWave communications with very high data rates (and thus very small symbol duration), intersymbol interference (ISI) due to time dispersion in multipath propagation becomes significant. Orthogonal frequency-division multiplexing (OFDM) signals are relatively robust against ISI due to the reduced symbol rate in each of the subcarriers, and thus, it is a good candidate for mmWave communications. Although we use OFDM in our system model, our work is independent of any particular modulation schemes.

OFDM can be combined with a multiple access scheme such as Time Division Multiplex Access (TDMA) or Code Division Multiple Access (CDMA) for effective multiple access control [4]. OFDM-TDMA are straightforward: different users share the wireless medium in different time slots. Several combinations of OFDM and CDMA have been discussed in [5]. For RF oscillators at mmWave spectrum, it is very difficult to maintain a low level phase noise, which affects the signal in the frequency conversion operations, and results in higher bit error rate (BER) for effective communications. Different multiple access techniques, including OFDM/TDMA, direct sequence (DS)-CDMA, Multi-Carrier (MC)-CDMA, and MCDS-CDMA, have different sensitivities to phase noise. According to [6], MC-DS-CDMA is most robust against phase noise and multiple access interference (MAI). Therefore, we deploy MC-DS-CDMA as the medium access technique for the mmWave networks.

\section{B. Directional Antennae}

Because of the unique characteristics of $60 \mathrm{GHz}$ mmWave communications, i.e., small wavelength and high path loss due to severe oxygen absorption and atmospheric attenuation, it is highly desired to use directional antenna to achieve much higher antenna gain over a longer transmission range, by radiating transmission energy to the desired direction only [7]. There are two types of directional antennae [8]: conventional sectored/switched antenna array and adaptive antenna array. A sectored antenna array consists of a number of fixed beams that provide full coverage in azimuth. An adaptive antenna array is able to automatically adapt its radiation patterns by using beamforming technique that intelligently puts a main beam in the direction of the wanted signal and nulls in the directions of the interference and noise. Since the size of the antennae used for mmWave communications could be very small, it is feasible to deploy multiple antenna elements in a device to achieve directivity. In a mmWave WPAN with directional antennae, directivity and high path loss should result in a more efficient spectrum reuse and significant improvement in the network throughput. In addition, directional antennae are more energy efficient.

In the networking research community, a popular antenna model for directional antenna is the flat-top model [9], [10]: the antenna gain is a constant within the beamwidth and zero outside the beamwidth. Therefore, for a beam with beamwidth $\theta$, the antenna gain of the mainlobe is $G_{m}=2 \pi / \theta$, and that of sidelobe is $G_{s}=0$. A more realistic three-dimensional cone plus sphere model is proposed in [11], taking the effects of sidelobes into consideration. In this model, the antenna gain consists of a mainlobe of beamwidth $\theta$ and aggregated spherical sidelobes of beamwidth $2 \pi-\theta$ at the base of the mainlobe cone. Uniform gain is also assumed for simplicity in the cone plus sphere model. Since we consider all devices in a WPAN to be in a plane, we employ the cone plus circle model in a two-dimensional scenario and define the antenna gains of the mainlobe and sidelobe as $G_{m}=\eta \frac{2 \pi}{\theta}$ and $G_{s}=(1-\eta) \frac{2 \pi}{2 \pi-\theta}$, respectively, where $\eta$ is the antenna radiation efficiency.

\section{WPAN Network Architecture}

Since mmWave communications cannot penetrate walls, we consider devices randomly distributed in an $L \times L$ square room. IEEE 802.15.3 is the standard dedicated for high rate WPANs. According to IEEE 802.15.3, multiple devices form a piconet which is the basic network element. One device is selected as the piconet controller (PNC) that collects the global information of the piconet. Data transmissions in the piconet is based on the time-slotted superframe structure [12]. Considering most devices using directional antenna in mmWave WPANs, the centralized PNC is very useful for device/neighbor discovery. The PNC broadcasts beacons periodically to all directions which allow other devices to synchronize and determine their locations. All devices send channel time requests and their locations to the PNC, which schedules peer-to-peer communications accordingly. However, the scheduling algorithm is not specified in the standard, and it is our focus.

\section{EXCLUSIVE REGIONS}

Let $P_{R}$ denote the received signal power, $R$ the channel capacity (or the achievable data rate with an efficient transceiver design), $N_{0}$ the one-sided spectral density of white Gaussian noise, and $I$ the total interference power. According to the Shannon theory, $R=W \log _{2}(S I N R+1)$, where $S I N R=P_{R} /\left(N_{0} W+I\right)$. 
Consider a network with $N$ flows, $\left\{f_{i}, \mid i \in 0,1, \ldots, N\right\}$, requesting transmission times in a superframe with $N$ time slots. The distance between the transmitter and receiver of the $i$-th flow is $d_{i}$, and the distance between the transmitter of the $j$-th flow and the receiver of the $i$-th flow is $d_{j, i}$. The average transmitting power and receiving power of flow $f_{i}$ are denoted as $P_{T}(i)$ and $P_{R}(i)$, respectively. Using the free space path loss model, $P_{R}(i)$ can be calculated as $P_{R}(i)=G_{T}(i) G_{R}(i)\left(\frac{\lambda}{4 \pi d_{i}}\right)^{2} P_{T}(i)$, where $G_{T}(i)$ and $G_{R}(i)$ are the antenna gains of the transmitter and receiver, respectively. Considering signal dispersion over distance, the average received signal power is modeled as

$$
P_{R}(i)=k_{1} G_{T}(i) G_{R}(i) d_{i}^{-\alpha} P_{T}(i),
$$

where $k_{1} \propto(\lambda / 4 \pi)^{2}$ is a constant coefficient dependent on the wavelength $\lambda$, and $\alpha$ is the path loss exponent dependent on the propagation environment and usually takes the value between 2 to 6 [13]. Assume that $G_{T}(i), G_{R}(i)$ and $\alpha$ are constant, and all devices use the same transmission power.

If only one flow is allowed to transmit at a time, i.e., flows are transmitted in a TDMA fashion, the average data rate of the $i$-th flow during the $N$ slots, $R_{i}$, is given by

$$
R_{i}=\frac{k_{2} W}{N} \log _{2}\left(\frac{k_{1} G_{T}(i) G_{R}(i) P_{T}(i) d_{i}^{-\alpha}}{N_{0} W}+1\right)
$$

where $k_{2}$ is a coefficient related to the efficiency of the transceiver design. If all flows can be transmitted simultaneously in all slots, i.e., flows are transmitted in a CDMA fashion, the achievable data rate, $R_{i}^{\prime}$, of the $i$-th flow is given by

$$
R_{i}^{\prime}=k_{2} W \log _{2}\left(\frac{k_{1} G_{T}(i) G_{R}(i) P_{T}(i) d_{i}^{-\alpha}}{N_{0} W+\sum_{i \neq j} I_{j, i}}+1\right)
$$

where $I_{j, i}$ is the interference power between the transmitter of the $j$-th flow and the receiver of the $i$-th flow. Assume the cross correlation between any two concurrent transmissions is constant, $G_{j, i}=G_{0}, \forall j \neq i$. The interference power is $I_{j, i}=k_{1} G_{0} G_{T}(j) G_{R}(i) P_{T}(j) d_{j, i}^{-\alpha}$.

To compare $R$ and $R^{\prime}$, we consider two cases separately. First, if SINR $<1$, the achieved data rate can be approximated as

$$
k_{2} W \log _{2}(\mathrm{SINR}+1) \approx k_{2} W \times \mathrm{SINR} \log _{2} e .
$$

With the approximation, from (2) and (3), a sufficient condition to ensure that $R_{i}^{\prime} \geq R_{i}$ is $I_{j, i} \leq N_{0} W, \quad \forall j \neq i$, i.e., the average interference level from any other flow should be less than the background noise ${ }^{1}$. Thus, if we allow flows with mutual interference less than that of the background noise to transmit simultaneously, the throughput of each flow can be higher than that of serial TDMA transmissions.

Second, if SINR $\geq 1$, the approximation in (4) may not hold. Nevertheless, the previous derived sufficient condition can still ensure that $R_{i} \leq R_{i}^{\prime}$. This is because $\log _{2}(x / N+$ 1) $\geq(1 / N) \log _{2}(x+1), \quad \forall x \geq 1, N \geq 1$. If $I_{j, i} \leq N_{0} W$,

\footnotetext{
${ }^{1}$ The necessary and sufficient condition to ensure that $R_{i}^{\prime} \geq R_{i}$ is $\sum_{j \neq i} I_{j, i} \leq(N-1) W N_{0}$, where flow $j$ is scheduled to transmit concurrently with flow $i$. The sufficient condition given in the main text is more conservative, but it allows to design much simpler and practically more feasible scheduling algorithms.
}

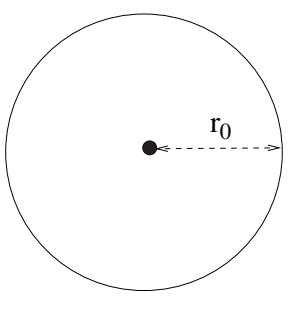

(a) Omni - Omni

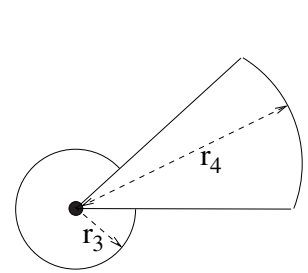

(c) Omni - Directional

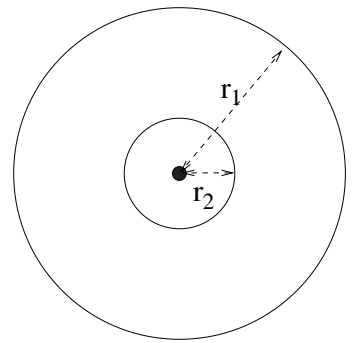

(b) Directional - Omni

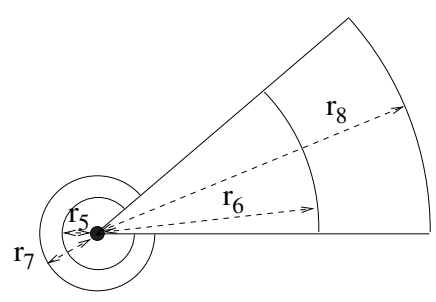

(d) Directional - Directional
Fig. 1. Exclusive regions for omni-directional and directional antennae.

$R_{i}^{\prime} / R_{i} \geq 1 / N \log _{2}(\mathrm{SNR}+1) / \log _{2}(\mathrm{SNR} / N+1) \geq 1$. Thus, the derived sufficient condition is still applicable.

Assume that the noise power spectrum is constant. To ensure that the interference power is less than the noise, we should not allow any interferer inside an ER around the receiver. In other words, an interferer should be at least $r(i)$ away from the receiver of the $i$-th flow, where $r(i)$ is given as

$$
r(i)=\left(\frac{k_{1} G_{0} G_{T}(j) G_{R}(i) P_{T}(j)}{N_{0} W}\right)^{1 / \alpha} .
$$

The ERs are determined by the types of transmitting and receiving antennae, i.e., omni- or directional. In the following, we consider four cases in a two-dimensional plane, and the results obtained can also be extended to three-dimensional space.

\section{Case 1: Omni-antenna to Omni-antenna}

In this case, both the transmitters and receivers use omniantennae, $G_{T}(i)=G_{R}(i)=1, \forall i \in 1,2, \ldots, N$. The interference between flows $j$ and $i$ is $I_{j, i}=k_{1} G_{0} P_{T}(j) d_{j, i}^{-\alpha}$. Assume all transmitters use the same power $P$ for transmission. To ensure that the interference from each interfere to be less than the noise, all interfering sources should be at least $r_{0}$ away from the receiver of the $i$-th flow $\left(d_{j, i} \geq r_{0}\right)$, where $r_{0}$ is given by

$$
r_{0}=\left(\frac{k_{1} G_{0} P}{N_{0} W}\right)^{1 / \alpha} .
$$

Therefore, the ER is a circle centered at the receiver, with radius $r_{0}$, as shown in Fig. 1 (a).

\section{Case 2: Directional-antenna to Omni-antenna}

In this case, the transmitter antennae are directional and the receiver antennae are omni-antennae $\left(G_{R}(i)=1\right)$. The directional antenna pattern consists of a mainlobe of gain $G_{T_{M}}$ with beamwidth $\theta$ and a sidelobe of gain $G_{T_{S}}$ with beamwidth $2 \pi-\theta$. 
As shown in Fig. 1(b), if a receiver is inside the radiation angle of an interferer, the interference is $I_{j, i}=k_{1} G_{0} G_{T_{M}} P d_{j, i}^{-\alpha}$. Thus, an interferer should be outside the circle centered at the receiver with radius $r_{1}$ :

$$
r_{1}=\left(\frac{k_{1} G_{0} G_{T_{M}} P}{N_{0} W}\right)^{1 / \alpha}
$$

If a receiver is outside the radiation angle of an interferer, we have $I_{j, i}=k_{1} G_{0} G_{T_{S}} P d_{j, i}^{-\alpha}$, and the ER is a circle with radius $r_{2}$ :

$$
r_{2}=\left(\frac{k_{1} G_{0} G_{T_{S}} P}{N_{0} W}\right)^{1 / \alpha} .
$$

\section{Case 3: Omni-antenna to Directional-antenna}

When the receiver antennae are directional and the transmitter antennae are omni-directional, the exclusive region in this case is a sector of a circle centered at the receiver with radius $r_{4}$ plus a sector with radius $r_{3}$ and angle $2 \pi-\theta$, as shown in Fig. 1(c), where $\theta$ is the beamwidth of the directional antenna of the receiver.

Let $G_{R_{M}}$ be the antenna gain of the receiver within the beamwidth of $\theta$, and $G_{R_{S}}$ the gain outside the beamwidth. If an interferer is located within the beamwidth of a receiver's antenna, $I_{j, i}=k_{1} G_{0} G_{R_{M}} P d_{j, i}^{-\alpha}$, and the interferer should be at least $r_{3}$ away from the receiver:

$$
r_{3}=\left(\frac{k_{1} G_{0} G_{R_{M}} P}{N_{0} W}\right)^{1 / \alpha} .
$$

Otherwise, $I_{j, i}=k_{1} G_{0} G_{R_{S}} P d_{j, i}^{-\alpha}$ and the interferer should be at least $r_{4}$ away from the receiver:

$$
r_{4}=\left(\frac{k_{1} G_{0} G_{R_{S}} P}{N_{0} W}\right)^{1 / \alpha}
$$

\section{Case 4: Directional-antenna to Directional-antenna}

When both the transmitter and receiver antennae are directional, the ER contains four zones. If an interferer is located within the beamwidth of the receiver, and the receiver is also within the beamwidth of the interferer, the interferer should be at least $r_{8}$ away from the receiver:

$$
r_{8}=\left(\frac{k_{1} G_{0} G_{T_{M}} G_{R_{M}} P}{N_{0} W}\right)^{1 / \alpha} .
$$

Therefore, the first ER zone is a cone with angle $\theta$ and radius $r_{8}$.

If an interferer is within the radiation angle of the receiver, but the receiver is outside the radiation angle of the interferer, the second ER zone is a cone with angle $\theta$ and radius $r_{6}$ :

$$
r_{6}=\left(\frac{k_{1} G_{0} G_{T_{S}} G_{R_{M}} P}{N_{0} W}\right)^{1 / \alpha} .
$$

If an interferer is outside the radiation angle of the receiver with its radiation beamwidth toward the receiver, the third ER zone is a sector with angle $2 \pi-\theta$ and radius $r_{7}$ :

$$
r_{7}=\left(\frac{k_{1} G_{0} G_{T_{M}} G_{R_{M}} P}{N_{0} W}\right)^{1 / \alpha} .
$$

If both the interferer and the receiver are outside of each other's radiation beamwidth, the last ER zone is a sector with angle $2 \pi-\theta$ and radius $r_{5}$ :

$$
r_{5}=\left(\frac{k_{1} G_{0} G_{T_{S}} G_{R_{S}} P}{N_{0} W}\right)^{1 / \alpha} .
$$

The four ER zones for this case are shown in Fig. 1 (d).

\section{REX SCHEDULING SCHEME}

\section{A. REX Scheme}

It is shown in Sec. III that concurrent transmissions are more favorable than serial TDMA transmissions if all interfering devices are sufficiently far apart, i.e., outside the ERs of the other receivers. In other words, network throughput can be improved by exploiting the spatial reuse of the wireless channel for concurrent transmissions. With a random network topology, the optimal scheduling problem for concurrent transmissions is known to be NP-hard [2], [3]. Unlike the traditional scheduling problems, each flow's throughput per time slot in mmWave WPANs is unknown before the scheduling decision, and it depends on network topology, user deployment, transmission power, cross-correlations of interfering signals, and the scheduling decision itself. If $P \neq N P$, there is no polynomial time algorithm to optimize the scheduling decision.

In the following, we propose REX, a randomized ER based scheduling scheme for a centralized mmWave WPAN, with computational complexity $O\left(N^{2} \log N\right)$ to allocate a time slot. We consider a WPAN with $N$ active flows requesting transmissions. The PNC has the global information of the WPAN, e.g., the number of active flows, and the location information of all devices, etc., based on which the PNC schedules peer-to-peer transmissions for active flows ${ }^{2}$. Denote the set of all active flows as $S\{N\}$ of $N$ elements. A subset of flows $\gamma_{i} \subset S\{N\}$ contains the flows scheduled in slot $i$ that satisfy the conditions favoring concurrent transmissions, as derived in (6)-(14). Denote $F S$ the set of scheduled flows in $S\{N\}$ and $T_{a}(j)$ the number of slots allocated to flow $j$. Initially, $F S=\gamma_{i}=N U L L$ and $T_{a}=0$ for all flows in any slot. The proposed REX scheme is as follows.

- Step 1: Randomly choose one flow with the minimum $T_{a}$ and schedule it in slot $i$ (initially, $i=1$ for the first slot). Add this flow to the subsets $\gamma_{i}$. If the flow is not included in $F S$, add it to $F S$;

- Step 2: Check all the remaining active flows in the set $S\{N\}-\gamma_{i}$ for concurrent transmission conditions as derived in (6)-(14), starting from the flow with the smallest $T_{a}$. If any flow satisfies the concurrent transmission condition, i.e., the new flow and the flows in set $\gamma_{i}$ are mutually outside each other's exclusive regions, add it to $\gamma_{i}$ and increase $T_{a}$ of the flow by one. If this flow is not included in $F S$, add it to $F S$;

- Step 3: Increase the slot number $i$ by one and sort flows according to $T_{a}$ in ascending order;

- Step 4: Repeat Steps 1-3 until all flows are scheduled, $F S=S\{N\}$.

The procedure can also be repeated until the requirements of all active flows are fully satisfied. It is worth noting that although sorting flows according to their $T_{a}$ in step 2 will increase the computational complexity by $O(N \log N)$, it is essential for maintaining fairness among flows. If we search

\footnotetext{
${ }^{2}$ In WPANs, the mobility is typically low, e.g., $\leq 1 \mathrm{~m} / \mathrm{s}$, and the superframe duration is less than $100 \mathrm{~ms}$. Thus, the node movement is normally less than $0.1 \mathrm{~m}$ during the superframe duration. Such small change in location will not significantly affect the received power and interference power level, and it is acceptable to ignore mobility for scheduling decision.
} 
flows in a deterministic sequence for slot allocation, those flows with smaller sequence number are more likely to be scheduled in $\gamma_{i}$. This will cause serious unfairness problem, as shown in the simulation results in a later section. With the searching sequence used in Step 2, the maximum access delay of all flows can be bounded.

The results of whether two flows are mutually exclusive can be saved in a look-up table to reduce the execution time of REX. Due to low mobility in WPANs, the frequency of updating this table is low.

\section{B. Average Number of Concurrent Transmissions}

Given the number of active users in an area, what is the number of flows that can transmit simultaneously under the constraint of the ER condition? Since network topology and user deployment drastically affect the network performance, we focus on the expected number of concurrent transmissions, which is general and independent of network topology and user deployment.

Consider an $L \times L$ square room containing $N$ active flows, with $N$ transmitters and $N$ receivers randomly deployed. Define $P(k, n)$ as the probability that only $k$ flows satisfy the ER condition and can be scheduled for concurrent transmissions, after checking the first $n \leq N$ flows one by one. Without loss of generality, we check flows in ascending order $1,2, \ldots, N$. The first flow $f_{1}$ will be scheduled for transmission in the set $\gamma$, and we have $P(1,1)=1$. Flow $f_{2}$ will be added to $\gamma$ if it does not conflict with flow $f_{1}$. Define $Q$ as the probability of a transmitter lying outside an ER of a receiver. The probability that a flow does not conflict with another flow is $Q^{2}$, because both transmitters should be outside the ERs of the other receivers. Accordingly, the probability that two flows do not satisfy the ER condition is $1-Q^{2}$. Therefore, in the two-flow case, we have $P(2,2)=Q^{2}$ and $P(1,2)=1-Q^{2}$. After we check the first $n$ flows, there are $k$ flows in $\gamma$ if a) there are $k-1$ flows in $\gamma$ when we check the first $n-1$ flows, and the $n$-th flow does not conflict with the other $k-1$ flows in $\gamma$; or b) there are $k$ flows in the set when we check the first $n-1$ flows, and the $n$-th flow conflicts with one of the $k$ flows in $\gamma$. The probability that a flow does not conflict with any of the other $k-1$ flows is $Q^{2(k-1)}$.

$$
\begin{aligned}
P(k, n)=\quad & P(k-1, n-1) Q^{2(k-1)} \\
& +P(k, n-1)\left(1-Q^{2 k}\right) \quad \text { for } k<n .
\end{aligned}
$$

If, among the $n$ flows, only the first flow can be added in $\gamma$, implying that the following $n-1$ flows do not satisfy the ER condition, we have

$$
P(1, n)=\left(1-Q^{2}\right)^{n-1} \quad \text { for } k=1 .
$$

Another extreme case is that all $n$ flows can be scheduled concurrently, which means that none of the flows conflicts with the remaining $n-1$ flows,

$$
P(n, n)=\left(Q^{n-1}\right)^{n} \quad \text { for } k=1 .
$$

Given the initial values of $P(1,1), P(1,2)$ and $P(2,2)$, we can iteratively obtain $P(k, N)$ as a function of $Q$ for $\forall k, 1 \leq$

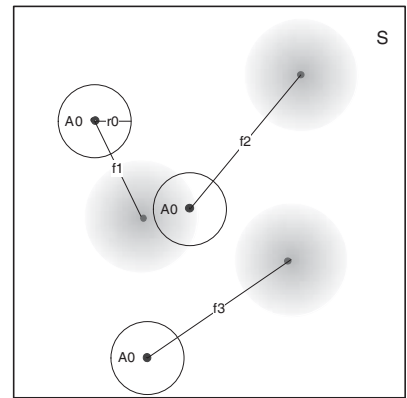

(a) Omni-Omni

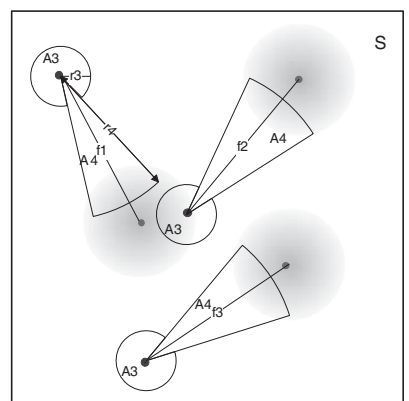

(c) Omni-Directional

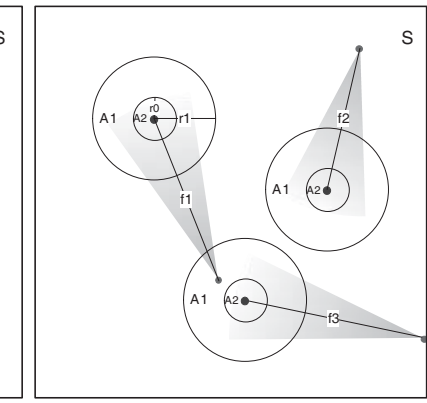

(b) Directional-Omni

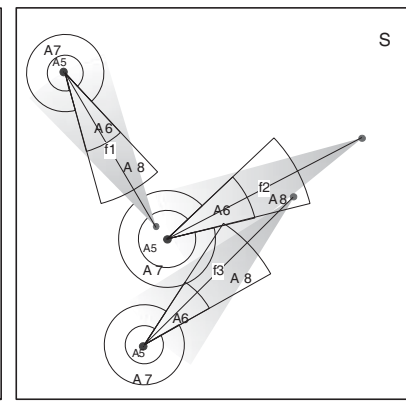

(d) Directional-Directional
Fig. 2. Concurrent transmissions in WPANs.

$k \leq N$. The expected number of concurrent transmissions is

$$
E[C T]=\sum_{k=1}^{N} k P(k, N) .
$$

To obtain $E[C T]$, we need to know $Q$. Let the size of the ER of a receiver be $A$, and total area $S=L^{2}$. As shown in Fig. 2, with each device randomly deployed in the room, an interferer of one flow is outside the ER of the receiver of another flow with probability $Q=1-A / S$. Since the ER region and $Q$ are related to the types of antennae used, in the following, we derive $Q$ by considering the four cases shown in Fig. 1.

\section{Case 1: Omni-antenna to Omni-antenna}

In case 1 , the ER is a circle with radius $r_{0}$ and $A_{0}=\pi r_{0}^{2}$, as shown in Fig. 2(a). The probability that an interferer is outside the ER of a receiver is given by

$$
Q_{1}=1-\frac{A_{0}}{S}=1-\frac{\pi r_{0}^{2}}{S}, \text { for } r_{0}<<L .
$$

\section{Case 2: Directional-antenna to Omni-antenna}

Due to the omni- receivers and directional transmitters, the ER in case 2 contains two zones, a circle with radius $r_{1}$ and another circle with radius $r_{2}$, as shown in Fig. 2(b). Accordingly, the areas of the two zones are $A_{1}=\pi r_{1}^{2}$ and $A_{2}=\pi r_{2}^{2}$. If a receiver is within the radiation angle of an interferer with probability $\theta / 2 \pi$, the interferer is outside the first ER zone $\left(A_{1}\right)$ with probability $1-A_{1} / S$. Similarly, if a receiver is outside the radiation angle of an interferer with probability $1-\theta / 2 \pi$, the interferer is outside the second ER zone $\left(A_{2}\right)$ with probability $1-A_{2} / S$. Therefore, the probability that an interferer is outside the ER of a receiver is given by

$$
Q_{2}=1-\frac{\pi r_{2}^{2}}{S}+\frac{r_{2}^{2} \theta}{2 S}-\frac{r_{1}^{2} \theta}{2 S}, \text { for } r_{1}, r_{2}<<L
$$




\section{Case 3: Omni-antenna to Directional-antenna}

The ER in case 3 contains two exclusive zones, one sector with radius $r_{3}$ and angle $2 \pi-\theta$, and the other sector with radius $r_{4}$ and angle $\theta$, as shown in Fig. 2(c). The areas of the two sectors are $A_{3}=\pi r_{3}^{2}(1-\theta / 2 \pi)$ and $A_{4}=\pi r_{4}^{2}(\theta / 2 \pi)=$ $\theta r_{4}^{2} / 2$. Note that the two areas are exclusive to each other, i.e., $A_{3} \cap A_{4}=N U L L$. Thus, an omni- interferer is outside the ER of a directional receiver if it is in neither $A_{3}$ nor $A_{4}$, and the probability is given by

$$
Q_{3}=1-\frac{\pi r_{3}^{2}}{S}+\frac{r_{3}^{2} \theta}{2 S}-\frac{r_{4}^{2} \theta}{2 S} \text { for } r_{3}, r_{4}<<L .
$$

If the antenna gain of the directional transmitter in case 2 equals that of the directional receiver in case $3\left(G_{T_{M}}=\right.$ $G_{R_{M}}$ ), we have $r_{2}=r_{3}$ and $r_{1}=r_{4}$ from (7)-(10), and thus $Q_{2}=Q_{3}$ from (20) and (21). Accordingly, from (18), the expected number of concurrent transmissions in case 2 equals that in case 3 if $G_{T_{M}}=G_{R_{M}}$.

\section{Case 4: Directional-antenna to Directional-antenna}

The ER in this case contains four zones, as shown in Fig. 2(d). The first zone $A_{5}$ is a sector with radius $r_{5}$ and angle $2 \pi-\theta$ and the second zone $A_{6}$ is a sector with radius $r_{6}$ and angle $\theta$. The areas of these two exclusive zones are $A_{5}=\pi r_{5}^{2}(1-\theta / 2 \pi)$ and $A_{6}=\pi r_{6}^{2}(\theta / 2 \pi)$. If a receiver is outside the radiation angle of an interferer with probability $1-\theta / 2 \pi$, the interferer is outside the ER zones of the receiver ( $A_{5}$ and $A_{6}$ ) with probability $1-\left(A_{5}+A_{6}\right) / S$. Similarly, if a receiver is within the radiation angle of an interferer with probability $\theta / 2 \pi$, the interferer is outside the ER zones of the receiver $\left(A_{7}\right.$ and $\left.A_{8}\right)$ with probability $1-\left(A_{7}+A_{8}\right) / S$, where $A_{7}=\pi r_{7}^{2}(1-\theta / 2 \pi)$ and $A_{8}=\pi r_{8}^{2}(\theta / 2 \pi)$. Therefore, we have

$$
Q_{4}=\left(1-\frac{A_{7}+A_{8}}{S}\right) \frac{\theta}{2 \pi}+\left(1-\frac{A_{5}+A_{6}}{S}\right)\left(1-\frac{\theta}{2 \pi}\right),
$$

for $r_{5}, r_{6}, r_{7}, r_{8}<<L$.

Edge Effect - If the areas of ER zones are relatively large compared to the room area or the device is located near the edge of the room, it is likely that some parts of the ER zones will be outside the room, and this is referred to as "edge effect". Using case 1 as an example, if the receiver is at the corner of the square room, we have $A^{\prime}=\pi r_{0}^{2} / 4<A$. The actual probability that a random interferer is within the ER of a receiver should be $A^{\prime} / S \leq A / S$. Thus, the developed analytical model without considering the edge effect may result in a conservative estimation of $E[C T]$. To mitigate the edge effect, we set a constraint for the area of ERs such that $A_{i} \leq S$ and $r_{i} \leq \sqrt{2} L, \forall i, i=0, \ldots, 8$, in all cases.

\section{Performance Evaluation}

We calculate the analytical results using Maple 10 [14] and compare the network performance of the proposed REX scheme with TDMA through extensive simulations using $\mathrm{C}$. The network is setup in a $10 \times 10 \mathrm{~m}^{2}$ area, with 20 to 80 active flows deployed in the area. The high dense scenarios can be in future conference rooms, shopping malls, expo rooms, etc. All flows use the transmission power of $10 \mathrm{~mW}$, and $k_{1}$ is $-51 \mathrm{~dB}$. Let $N_{0}=-114 \mathrm{dBm} / \mathrm{MHz}, W=500 \mathrm{MHz}$, and $G_{0}=10^{-2} . k_{2}$ is set to one for calculation simplicity. We

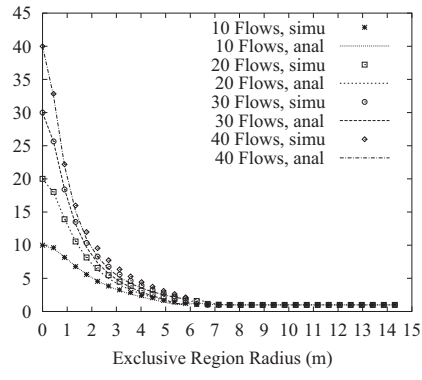

(a) case 1

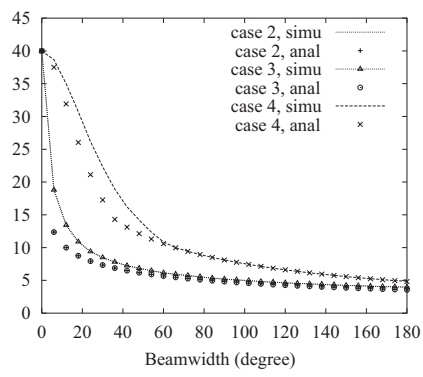

(b) cases 2 - 4
Fig. 3. Number of concurrent transmissions $(\alpha=4)$.

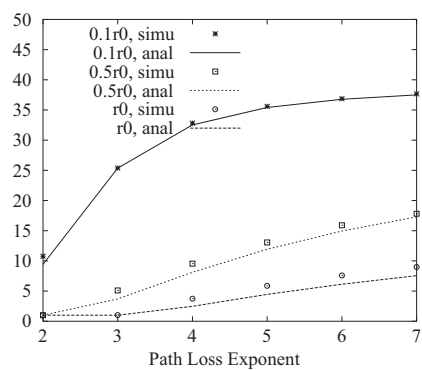

(a) case 1

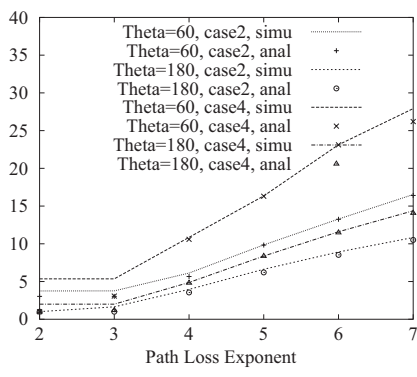

(b) cases 2 - 4
Fig. 4. Number of concurrent transmissions vs. path loss exponent.

repeat the simulation 500 times with different random seeds and calculate the average value.

\section{A. Spatial multiplexing gain}

We first apply the ideal flat-top antenna model for the directional antenna: $\eta=1, G_{m}=2 \pi / \theta$ and $G_{s}=0$. As shown in Figs. 3 (a) and (b), since the ER size increases with the ER radius $r$ or the beamwidth $\theta$, fewer flows can be scheduled for concurrent transmissions. The relationship between the number of concurrent transmissions and path loss exponent $\alpha$ is shown in Fig. 4. According to (6)-(14), higher path loss exponent results in a smaller $r$ and ER size, and thus a more aggressive spatial reuse can be achieved. When $\alpha$ or $\theta$ is small, (e.g., $\alpha \leq 3$ or $\theta \leq 30$ degree), the ER radius could be very large and the edge effect becomes significant, so our analysis is more conservative due to the edge effect. Overall, simulation results validate the accuracy of our analysis.

The spatial multiplexing gain, defined as the ratio of network throughput with the proposed REX scheme to that with serial TDMA, is shown in Fig. 5. For case 1 (omni-to-omni), the sufficient condition to favor concurrent transmissions is to set the ERs as circles with radius $r_{0}=4.47 \mathrm{~m}$ centered at each receiver, as derived in (6). Fig. 5 (a) shows that with ER size set to $4.47 \mathrm{~m}$, the network throughput can be enhanced by more than 4 times, and the highest network throughput can be achieved if the ER is around $3 \mathrm{~m}$. Although more flows satisfy the concurrent transmission condition with a smaller ER size, higher interference among these flows may limit the network throughput. By setting the ER size to $r_{0}$, we can ensure that the spatial multiplexing gain is always greater than one. When the ER size is large enough to forbid any concurrent transmission, the performance of the proposed scheme is the same as that 


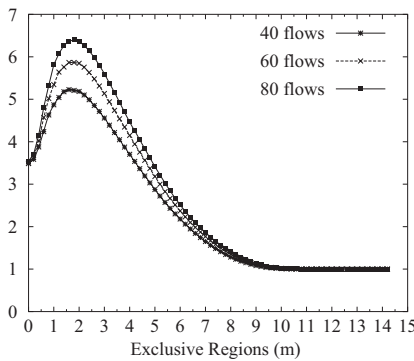

(a) case 1

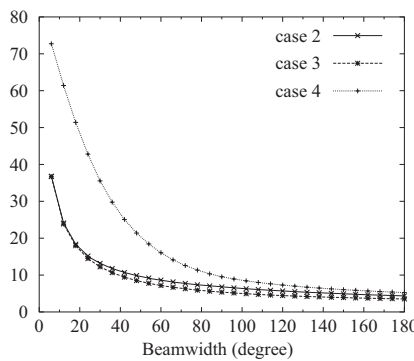

(b) cases $2-4$

Fig. 5. Spatial multiplexing gain.

of TDMA and the spatial multiplexing gain equals 1 . The results demonstrate the effectiveness and efficiency of the REX scheme.

Fig. 5(b) shows the spatial multiplexing gains obtained in cases 2-4. The ER size is set according to the $r_{1}$ to $r_{8}$ derived in (7) to (14). When $\theta=6$ degree, the ERs are set as a circle and a sector with radius $12.4 \mathrm{~m}$ for case 2 and case 3 , respectively. Most flows can be transmitted concurrently with such a small beamwidth and the spatial multiplexing gains are as high as 38 in case 2 and case 3 , and 73 in case 4 . The results shows that higher spatial multiplexing gain can be achieved with smaller beamwidth, because more flows satisfy the concurrent transmission conditions. We also observe that spatial multiplexing gain obtained in case 2 is slightly higher than that in case 3 , although the expected number of concurrent transmissions in these two cases are the same, as shown in Fig. 3 (b). This is because directional transmitters cause less interference to other concurrent transmissions than omnitransmitters. The spatial multiplexing gain is further improved when both receiver and transmitter use directional antennae. In all cases, the proposed REX scheme can achieve significant spatial multiplexing gains.

\section{B. Impact of radiation efficiency}

The flat-top model assumes the antenna radiation efficiency is 1 and the sidelobe level (SLL) is zero. In reality, the antenna gain is a product of the antenna directivity gain and the radiation efficiency $\eta<1$, and the sidelobe is non-zero. Therefore, we investigate the expected number of concurrent transmissions in case 4 with various values of $\eta$. The results are shown in Fig. 6, with the cone plus circle model introduced in Sec. II-B. The antenna gain within the beamwidth deceases by a factor of $\eta$, which results in an ER sector with smaller radius $r_{8}$. However, other ER zones (sectors with radius $r_{5}, r_{6}$, and $r_{7}$ ) are non-zero when the sidelobes are considered. Take $\theta=40$ degree for example. The antenna gain is 9 when $\eta=1$, and decreases to 8.1 when $\eta=0.9$. Without considering the sidelobe, the ER for $\eta=1$ is a sector of a circle with radius $r_{8}=13.4 \mathrm{~m}$. When $\eta=0.9$, the ER contains four zones, with $r_{5}=1.5 \mathrm{~m}, r_{6}=r_{7}=4.5 \mathrm{~m}$ and $r_{8}=12.7 \mathrm{~m}$, as shown in Fig. 1 (d). Obviously, the areas of the four ER zones for $\eta=0.9$ are larger than those for $\eta=1$. According to (19)(22), the probability, $Q$, that a transmitter is outside the ER of a receiver decreases when the ER areas increase, and thus fewer concurrent transmissions can be scheduled. As shown in

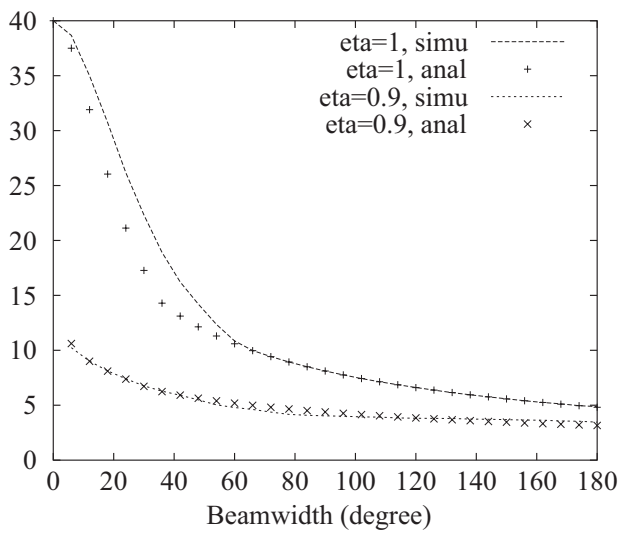

Fig. 6. Number of concurrent transmissions, case 4.

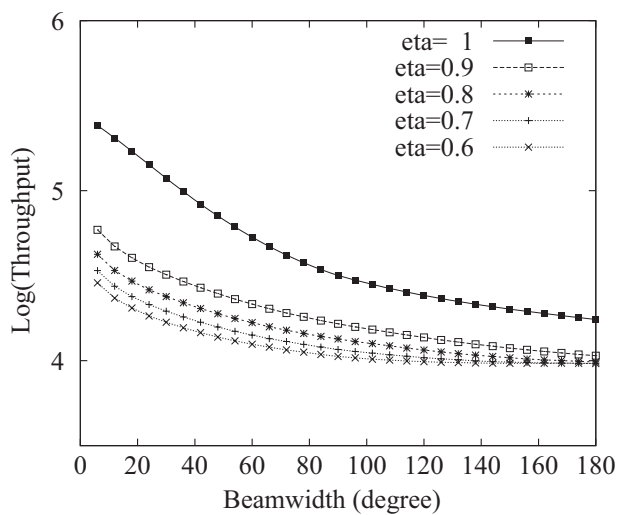

Fig. 7. Throughput with different antenna efficiency, case 4.

Fig. 6, when $\eta$ is reduced from 1 to 0.9 , the expected number of concurrent transmissions decreases significantly due to the increased ER areas. The base 10 logarithmic throughput in case 4 is shown in Fig. 7. It can be seen that the throughput also decreases quickly when $\eta$ decreases.

\section{Fairness}

The fairness of REX and TDMA are first measured by Jain's fairness index [15], $\left(\sum x_{i}\right)^{2} /\left(N \sum x_{i}^{2}\right)$, in terms of the total number of time slots allocated to each flow. The fairness index in cases 1 to 4 are shown in Figs. 8. TDMA can always achieve perfect fairness since it allocates time slots evenly to all flows. If we schedule concurrent transmissions with a deterministic searching scheme, all 40 flows are examined in the ascending order of the flow number, the flows with smaller sequence numbers are more likely to be scheduled and vice versa. As shown in Fig. 8 (a), with the deterministic searching sequence, fairness degrades drastically when the number of flows increases, while the proposed REX scheduling scheme using sorted searching sequence can achieve better fairness, regardless of the number of flows. Similar tendency can be found for cases 2 to 4, as shown in Fig. 8 (b).

On the other hand, for fair resource allocation, it is desirable to maximize the minimal flow throughput among all competing flows. We compare the per-flow throughput of REX and TDMA, and the maximum and minimum per-flow throughputs are shown in Fig. 9. Among the 40 flows, when the beamwidth 


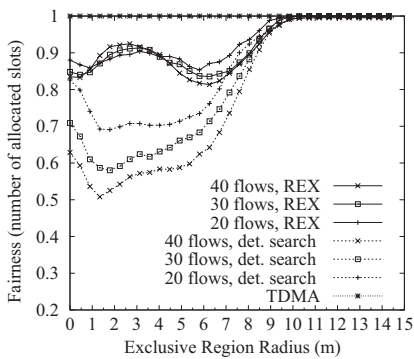

(a) case 1

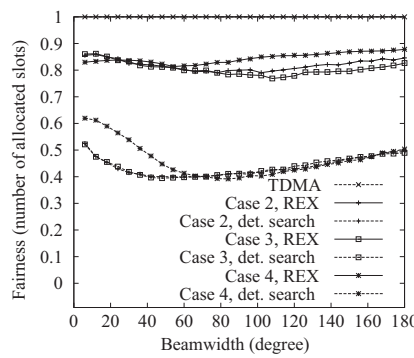

(b) cases $2-4$
Fig. 8. Fairness comparison w.r.t number of allocated slots.

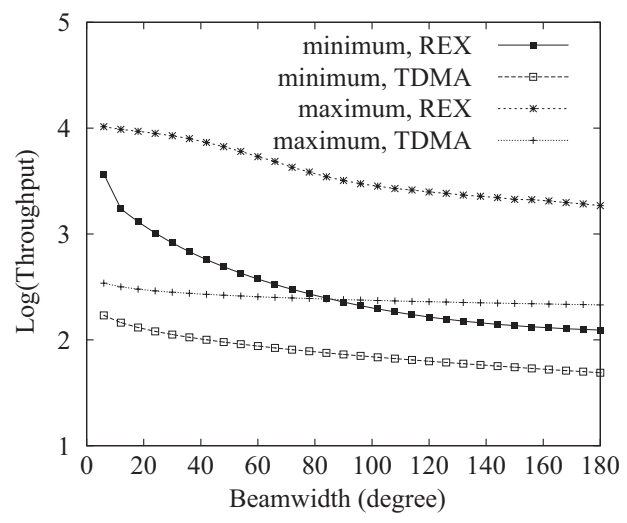

Fig. 9. Single flow throughput (case 4).

is less than 80 degrees, the minimal per-flow throughput with REX is even higher than the maximum per-flow throughput with TDMA; when the beamwidth is larger than 80 degrees, the minimal per-flow throughput with REX is still much higher than that with TDMA. Although Jain's fairness index of REX is lower than that of TDMA, the minimal per-flow throughput is significantly improved with the proposed scheme. Thus, the REX scheme achieves better Min-Max fairness and it is very desirable to improve user experience.

\section{RELATED WORK}

Optimizing resource allocation for wireless networks has been an active research topic because wireless resource is at a premium. Since wireless communication is broadcast in nature, flow throughput and network throughput are limited by interference and collisions. Contention graph is a general approach for scheduling in multihop wireless networks [2], [16], [17], in which collision occurs when two or more devices within a one-hop distance in connectivity graph transmit simultaneously; and links that do not interfere with each other can be scheduled for concurrent transmissions. In [18], to quantify wireless network capacity, a protocol model was proposed for communications without rate adaptation, and an exclusive region concept was given. Without rate adaptation, the ER in [18] is a function of distance between the transceivers, which is different from the ER we discussed.

On the other hand, with the advances of physical layer technologies, devices can tolerate a certain level of interference and adapt the transmission rate according to the received SINR. Such rate adaptation techniques are widely used, e.g., in IEEE 802.11, IEEE 802.16, and UWB, etc., and they provide great potential to improve the wireless resource utilization. In [19], a general model for joint scheduling, power allocation and routing optimization problem was proposed for UWB networks. It concluded that the optimal MAC protocol in an UWB network should be a combination of rate adaptation and mutual exclusion. However, due to high complexity, the scheduling scheme was not investigated in [19]. A resource management scheme for UWB networks was proposed in [20], which aimed to solve the near-sender-blocking problem and alleviate the negative effect of long acquisition time of UWB transmissions. In [21], the optimal scheduling problem for UWB networks supporting heterogeneous traffic was formulated as a utility maximization problem under the fairness constraint. To the best of our knowledge, there is little work on resource management issues in mmWave WPANs reported in the literature, considering the salient features of mmWave communications with directional antenna.

\section{CONCLUSION}

In this paper, we have obtained the sufficient conditions in terms of ERs to ensure that the concurrent transmission scheme can outperform the TDMA scheme, considering both omni- and directional antennae. In addition, we have proposed the REX scheduling scheme, and derived the average number of concurrent transmissions given the ER condition. By scheduling appropriate concurrent transmissions, significant spatial multiplexing gain can be achieved, especially for mmWave WPANs with directional antennae.

We have used the transceiver distance to estimate the average received signal strength and interference level without considering fast fading. This is because the transmissions scheduled in WPANs are peer-to-peer transmissions, and it is very costly to measure their instantaneous channel quality. Future research issues include considering power control, user mobility, and fast channel fading, proposing distributed scheduling algorithm, and extending the work to three dimensional space. On the other hand, the analysis of the average number of concurrent transmissions is not only important for mmWave based WPANs, but also reveals the important relationship between the ER condition and the spatial multiplexing property of wireless networks in general. The proposed analytical framework should be helpful in revealing the fundamental theoretical bounds of general wireless networks in the future.

\section{REFERENCES}

[1] S. M. Fulton, "IBM's WPAN chipset aims to replace high-def cables," Bluetooth. Available in http://www.tgdaily.com/2006/02/07/ibm_wpan _chipset_aims_to_replace_cables, Feb. 2006.

[2] S. Ramanathan, "A unified framework and algorithms for (T/F/C)DMA channel assignment in wireless networks," in Proc. IEEE Infocom'97, pp. 900-907, Apr. 1997.

[3] K. H. Liu, L. Cai, and X. Shen, "Exclusive-region based scheduling algorithms for UWB WPAN," IEEE Trans. Wireless Commun., vol. 7, no. 3, pp. 933-942, Mar. 2008.

[4] A. Klein and R. W. Brodersen, "Multiple access extensions of OFDM," Jan. 2000

[5] S. Hara and R. Prasad, "Overview of multicarrier CDMA," IEEE Commun. Mag., pp. 126-133, Dec. 1997.

[6] C. Garnier, L. Clavier, Y. Delignon, M. Loosvelt, and D. Boulinguez, "Multiple access for $60 \mathrm{GHz}$ mobile ad hoc network," in Proc. VTC Spring 2002, vol. 3, pp. 1517-1521, May 2002. 
[7] S. Roy, Y. C. Hu, D. Peroulis, and X. Y. Li, "Minimum energy broadcast using practical directional antennas in all-wireless networks," in Proc. IEEE Infocom'06, 2006.

[8] C. Balanis, Antenna Theory, Analysis and Design. Hoboken, NJ: John Wiley \& Sons, Inc., 1997.

[9] J. E. Wieselthier, G. D. Nguyen, and A. Ephremides, "Energy-limited wireless networking with directional antennas: the case of session-based multicasting," in Proc. IEEE Infocom'02, vol. 1, pp. 190-199, June 2002.

[10] I. Kang and R. Poovendran, "Power-efficient broadcast routing in adhoc networks using directional antennas: technology dependence and convergence issues," technical report, UWEETR-2003-0015, July 2003.

[11] R. Ramanathan, "On the performance of ad hoc networks with beamforming antennas," in Proc. ACM MobiHoc, pp. 95-105, Oct. 2001.

[12] IEEE 802.15.3 TG, "IEEE std 802.15.3 TM $^{T M}$ 2003: wireless medium access control (MAC) and physical layer (PHY) specifications for high rate wireless personal area networks (WPANs)," Sep. 2003.

[13] J. S. Davis, "Indoor wireless RF channels." Available in http://wireless.per.n1/reference/chaptr03/indoor.htm.

[14] A. Heck, Introduction to Maple (3rd ed.). New York: Springer-Verlag, 2003.

[15] R. Jain, A. Durresi, and G. Babic, "Throughput fairness index: an explanation," in ATM Forum Document Number: ATM Forum/990045, Feb. 1999.

[16] K. Sundaresan and R. Sivakumar, "A unified MAC layer framework for ad-hoc networks with smart antennas," in Proc. ACM MobiHoc, vol. 15, pp. 546-559, 2004.

[17] W. Wang and X. Liu, "A framework for maximum capacity in multichannel multi-radio wireless," in Proc. CCNC'06, vol. 2, pp. 720-724, Jan. 2006.

[18] P. Gupta and P. Kumar, "The capacity of wireless networks," IEEE Trans. Inf. Theory, vol. 46, no. 2, pp. 388-404, Mar. 2000.

[19] B. Radunovic and J. Le Boudec, "Optimal power control, scheduling, and routing in UWB networkss," IEEE J. Sel. Areas Commun., vol. 22, no. 7, pp. 1252-1270, Sep. 2004.

[20] H. Jiang, K. H. Liu, W. Zhuang, and X. Shen, "An effective resource management scheme for UWB networks with simultaneous transmissions," IEEE Trans. Wireless Commun., vol. 6, no. 8, pp. 3005-3015, Aug. 2007.

[21] K. H. Liu, L. Cai, and X. Shen, "Multi-class utility-based scheduling for UWB networks," IEEE Trans. Veh. Technol., vol. 57, no. 2, Mar. 2008.

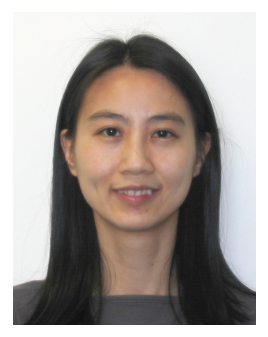

Lin X. Cai (S'09) received the B.Eng. degree in computer science from Nanjing University of Science and Technology, Nanjing, China, in 1996 and the M.A.Sc. degree in electrical and computer engineering from the University of Waterloo, Waterloo, Canada, in 2005. She is currently working toward a Ph.D. degree in the same field at the University of Waterloo. Her current research interests include network performance analysis and protocol design for multimedia applications over broadband wireless networks.

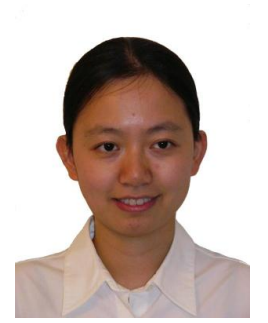

Lin Cai (S'00-M'06) received the M.A.Sc. and $\mathrm{Ph} . \mathrm{D}$. degrees (with Outstanding Achievement in Graduate Studies Award) in electrical and computer engineering from the University of Waterloo, Waterloo, Canada, in 2002 and 2005, respectively. Since July 2005, she has been an Assistant Professor in the Department of Electrical and Computer Engineering at the University of Victoria, British Columbia, Canada. Her research interests span several areas in wireless communications and networking, with a focus on network protocol and architecture design supporting emerging multimedia traffic over wireless, mobile, ad hoc, and sensor networks. She serves as the Associate Editor for IEEE TRANSACTIONS ON VEHICULAR TECHNOLOGY (2007- ), EURASIP JOURNAL ON WIRELESS COMMUNICATIONS AND NETWORKING (2006- ), and INTERNATIONAL JOURNAL OF SENSOR NETWORKS (2006- ).

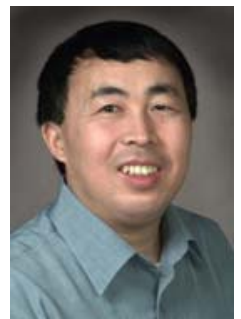

Xuemin (Sherman) Shen (F) received the B.Sc.(1982) degree from Dalian Maritime University (China) and the M.Sc. (1987) and Ph.D. degrees (1990) from Rutgers University, New Jersey (USA), all in electrical engineering. He is a Professor and University Research Chair, Department of Electrical and Computer Engineering, University of Waterloo, Canada. Dr. Shen's research focuses on resource management in interconnected wireless/wired networks, UWB wireless communications networks, wireless network security, wireless body area networks and vehicular ad hoc and sensor networks. He is a co-author of three books, and has published more than 400 papers and book chapters in wireless communications and networks, control and filtering. Dr. Shen served as the Technical Program Committee Chair for IEEE VTC'10, the Tutorial Chair for IEEE ICC'08, the Technical Program Committee Chair for IEEE Globecom'07, the General Co-Chair for Chinacom'07 and QShine'06, the Founding Chair for IEEE Communications Society Technical Committee on P2P Communications and Networking. He also serves as a Founding Area Editor for IEEE TRANSACTIONS ON WIRELESS COMMUNICATIONS; Editor-in-Chief for PeER-To-PeER Networking ANd Application; Associate Editor for IEEE TRANSACTIONS ON VEHICULAR TECHNOLOGY; COMPUTER NETWORKS; and ACM/WIRELESS NETwORKS, etc. He has also served as Guest Editor for IEEE JSAC, IEEE WIRELESS COMMUNICATIONS, IEEE COMMUNICATIONS MAGAZINE, and ACM MobILE NETwORKS AND ApPlications, etc. Dr. Shen received the Excellent Graduate Supervision Award in 2006, and the Outstanding Performance Award in 2004 and 2008 from the University of Waterloo, the Premier's Research Excellence Award (PREA) in 2003 from the Province of Ontario, Canada, and the Distinguished Performance Award in 2002 and 2007 from the Faculty of Engineering, University of Waterloo. Dr. Shen is a registered Professional Engineer of Ontario, Canada, an IEEE Fellow, and a Distinguished Lecturer of IEEE Communications Society.

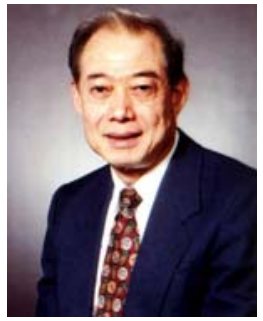

Jon W. Mark (LF) received the Ph.D. degree in electrical engineering from McMaster University in 1970. In September 1970 he joined the Department of Electrical and Computer Engineering, University of Waterloo, Waterloo, Ontario, where he is currently a Distinguished Professor Emeritus. He served as the Department Chairman during the period July 1984-June 1990. In 1996 he established the Centre for Wireless Communications (CWC) at the University of Waterloo and is currently serving as its founding Director. Dr. Mark had been on sabbatical leave at the following places: IBM Thomas J. Watson Research Center, Yorktown Heights, NY, as a Visiting Research Scientist (1976-77); AT\&T Bell Laboratories, Murray Hill, NJ, as a Resident Consultant (198283): Laboratoire MASI, Université Pierre et Marie Curie, Paris France, as an Invited Professor (1990-91); and Department of Electrical Engineering, National University of Singapore, as a Visiting Professor (1994-95).

$\mathrm{He}$ has previously worked in the areas of adaptive equalization, image and video coding, spread spectrum communications, computer communication networks, ATM switch design and traffic management. His current research interests are in broadband wireless communications, resource and mobility management, and cross domain interworking. He is a co-author of the text Wireless Communications and Networking, Prentice-Hall 2003. A Life Fellow of IEEE, Dr. Mark is the recipient of the 2000 Canadian Award for Telecommunications Research and the 2000 Award of Merit of the Education Foundation of the Federation of Chinese Canadian Professionals. He was an editor of IEEE Transactions on Communications (1983-1990), a member of the Inter-Society Steering Committee of the IEEE/ACM TRANSACTIONS ON NETWOR KING (1992-2003), a member of the IEEE Communications Society Awards Committee (1995-1998), an editor of WiRELESS NETwORKS (19932004), and an associate editor of TELECOMMUNICATION SySTEMS (19942004). 